\title{
New opportunities for managing acute and chronic lung infections
}

\section{William O. C. M. Cookson, Michael J. Cox and Miriam F. Moffatt}

Abstract | Lung diseases caused by microbial infections affect hundreds of millions of children and adults throughout the world. In Western populations, the treatment of lung infections is a primary driver of antibiotic resistance. Traditional therapeutic strategies have been based on the premise that the healthy lung is sterile and that infections grow in a pristine environment. As a consequence, rapid advances in our understanding of the composition of the microbiota of the skin and bowel have not yet been matched by studies of the respiratory tree. The recognition that the lungs are as populated with microorganisms as other mucosal surfaces provides the opportunity to reconsider the mechanisms and management of lung infections. Molecular analyses of the lung microbiota are revealing profound adverse responses to widespread antibiotic use, urbanization and globalization. This Opinion article proposes how technologies and concepts flowing from the Human Microbiome Project can transform the diagnosis and treatment of common lung diseases.

The airways and the lung are in a constant state of challenge from environmental microorganisms. A sedentary adult can inhale more than 10,000 litres of air per day $^{1}$, which washes over $40-80 \mathrm{~m}^{2}$ of lung surface $^{2,3}$. In cities, this air may contain up to 100,000 bacteria per litre 4 , a proportion of which are able to penetrate into the thoracic airways and beyond. By contrast, the gut has a surface area of $30 \mathrm{~m}^{2}$ (REF. 5), and microbial ingression is limited by gastric acid. Thus, it is not surprising that lung infections commonly affect human health. In the United Kingdom alone, 16 million patients of the National Health Service are treated with antibiotics each year for respiratory tract infections that include 260,000 episodes of community-acquired pneumonia $(\mathrm{CAP})^{6}$. Antibiotic prescription rates are higher in many other European countries than in the United Kingdom? In the United States, acute lung infection in the presence of underlying chronic obstructive pulmonary disease (COPD) is the most common reason for administering antimicrobial therapy to adults; this clinical setting plays a substantial role in driving antimicrobial resistance ${ }^{8}$.
Until recently, standard medical teaching held that the healthy lung is sterile $e^{9}$ and that pathogens invade an environment that is normally kept free of microorganisms by the host mucosa. However, the thoracic airways, which are the site of many infections, are now known to support a distinctive community with a density of bacteria similar to that of the small bowel ${ }^{10,11}$. The consideration of how diverse commensal microbial communities resist exogenous infection ${ }^{12-14}$ has had an enormous influence on understanding and treating bowel diseases $^{15}$, and it is timely now to reconsider received wisdom for the management of lung infections. Accessing the lower airways for routine microbial analyses typically involves invasive procedures such as fibre-optic bronchoscopy. These practical difficulties, and the low priority given to non-HIV lung disease by the Human Microbiome Project (HMP), mean that the technological and analytical advances of the HMP have been sparsely applied to the airways and the lung.

In this Opinion article, we review the most common pulmonary infective agents, with an emphasis on bacterial pathogens and their potential interactions with each other and airway commensals. We discuss the emergence of antibiotic multidrugresistant (MDR) organisms and highlight the potential for the global spread of pathogens through circulation in cities, schools and hospitals. We then suggest novel diagnostic and therapeutic possibilities for mitigating these serious problems. We propose that the opportunities to improve patient care are substantial and that a coordinated and collaborative international Lung Microbiome Project (to consider the collective genomes of the microorganisms that reside in the lung) together with systematic studies of individual organisms (the microbiota) will be of inestimable value to global health.

\section{The burden of lung infections}

Overt respiratory infections are the leading cause of death in developing countries and are associated with more than 4 million deaths annually ${ }^{16}$. In children under 5 years of age, pneumonia, which is usually associated with a bacterial infection, accounts for 1.3 million deaths annually and $18 \%$ of all-cause mortality ${ }^{17}$. Moreover, pneumonia kills far more adults than HIV or malaria ${ }^{16}$. Globally, lower respiratory infections result in the loss of 103,000 disability-adjusted life years, which means that lung infections are the largest cause of disease burden to humanity.

Acute infections can afflict healthy adults but are more common in the very young or very old or in those with underlying health problems. They usually affect the lung parenchyma (pneumonia), the airways of the lung (bronchitis) or both (bronchopneumonia) (FIG. 1). Less frequently, infections can spread to the linings of the lung, causing pleurisy and empyema. CAP differs from hospitalacquired pneumonia (HAP) and ventilator-associated pneumonia (VAP), which occur in patients who are sick or immunocompromised and result in high morbidity, mortality and costs ${ }^{18}$.

In contrast to acute infections, chronic lung infections are typically a consequence of underlying disease. For example, bronchiectasis describes dilations in the thoracic airways (FIG. 1), which can be caused by tuberculosis, scarring after 


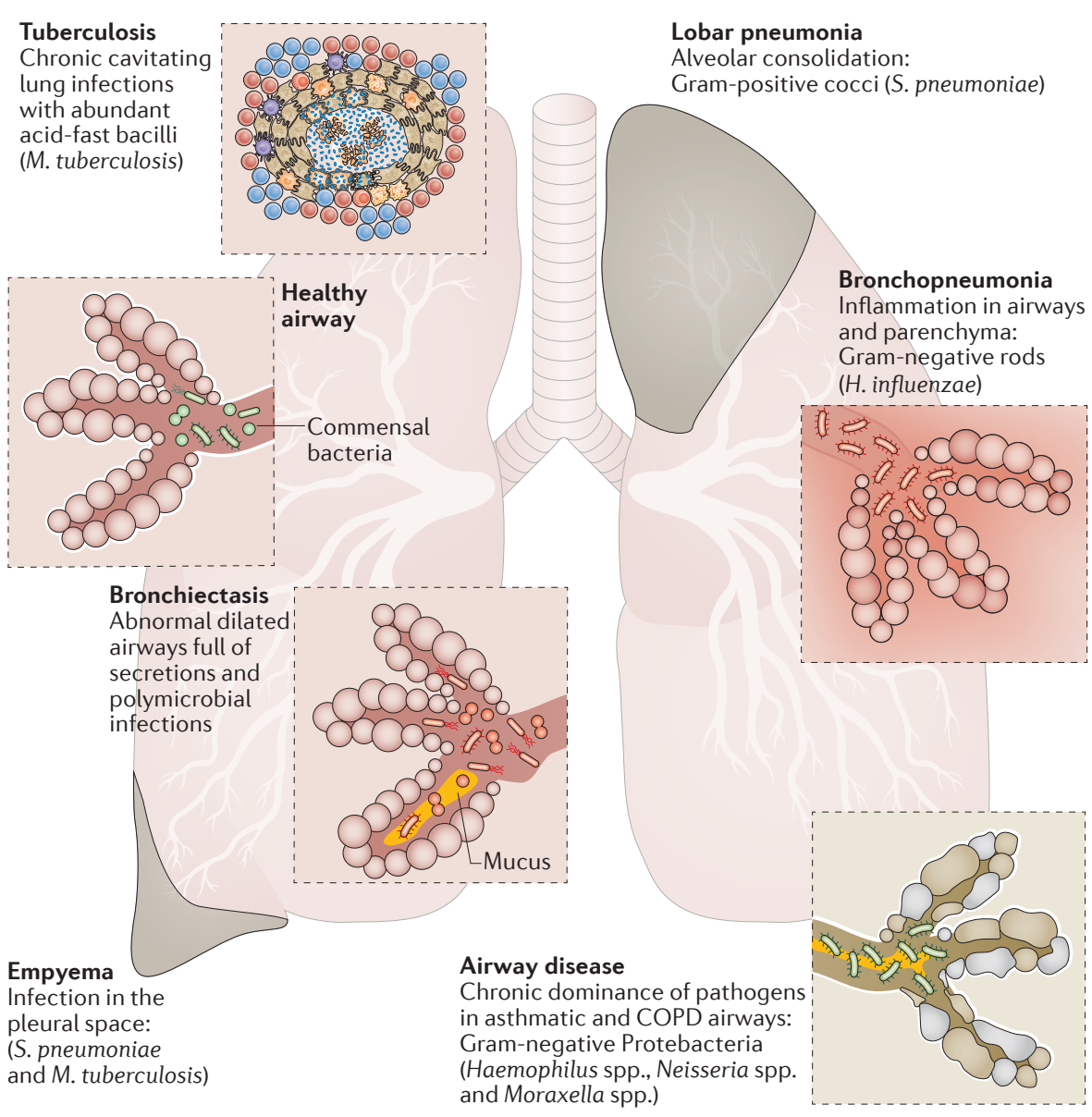

Figure 1 | Pulmonary infections associated with bacterial pathogens. The airways and lung can be affected in a number of different ways by bacterial infection, as shown clockwise starting from the top right: lobar pneumonia affects a whole lobe of the lung and may spill into the surrounding pleura (two thin layers of tissue that protect the lungs and allow them to move during breathing). Lobar pneumonia is typically caused by Gram-positive short chains of Streptococcus pneumoniae. Bronchopneumonia is centred in the bronchial tree and spreads into adjoining lung tissues; it is most commonly caused by the Gram-negative rods of Haemophilus influenzae. Asthma and chronic obstructive pulmonary disease (COPD) are diseases of the small airways, characterized by overgrowth of Proteobacteria (such as H. influenzae and Neisseria spp.) and the loss of normal commensal species. Empyema is an infection of the pleural space and is most commonly caused by S. pneumoniae. The chronic lung diseases bronchiectasis and cystic fibrosis are associated with chronic resistant infections with abnormal bronchial morphology; these infections are caused by $H$. influenzae, S. pneumoniae and opportunistic pathogens such as Staphylococcus aureus (a Gram-positive coccus) and Pseudomonas aeruginosa (a Gram-negative flagellate bacillus). Mycobacterium tuberculosis causes chronic consolidation and cavitation with invasion into draining lymph nodes and eventual systematic spread.

acute infection, primary ciliary dyskinesia, immune deficiencies and cystic fibrosis (in populations of European descent). Bronchiectasis from all causes results in the progressive production of thick, purulent secretions (sputum) and an advancing destruction of the airways and lung parenchyma.

Another underlying disorder that predisposes to infection is COPD, which is typified by inelastic constricted airways with or without loss of lung parenchyma (emphysema) and is usually the result of cigarette smoking. Chronic lung diseases such as COPD are all accompanied by periods of acute worsening of respiratory symptoms, known as exacerbations. The aetiology of exacerbations encompasses host immune responses as well as complex changes in the viral, fungal and bacterial communities ${ }^{19}$. In COPD, acute infective exacerbations cause airway inflammation and hasten disease progression ${ }^{20}$.

In other conditions, the relationship with infection is more complex. Asthma is a syndrome of intermittent airway inflammation that causes wheezing and shortness of breath. Notably, asthma affects 250-300 million individuals of all ages, races and ethnicities globally, and its prevalence is increasing alarmingly in the developing world ${ }^{21}$. Urbanization, with the loss of the microbial environment from traditional rural lifestyles, strongly increases susceptibility to disease ${ }^{22}$.

Exacerbations are often triggered by viral infections ${ }^{23}$. Asthmatic airways typically contain considerable numbers of bacterial pathogens ${ }^{24-28}$ (FIG. 1) and may also carry as yet unexplored fungi. However, a causal link between these organisms and disease is yet to be established.

\section{The microbiota of healthy lungs}

Pulmonary infections with pathogens take place in the presence of commensal lower-airway microbial communities (the microbiota; reviewed in REF. 29). In brief, the sequencing of variable regions of the $16 \mathrm{~S}$ rRNA gene has shown that healthy airways contain in the region of 100 operational taxonomic units (OTUs; as defined by sequence similarity), which are dominated by the Firmicutes (primarily Streptococcus spp. and Veillonella spp. OTUs) but also contain Bacteroidetes (mostly Prevotella spp.), Fusobacteria, Actinobacteria and Proteobacteria ${ }^{10,24-28}$. Many of these OTUs may represent facultative anaerobes that are recalcitrant to routine culture. The limited number of phyla and the consistency of the oropharyngeal and lower-airway microbiota between multiple individuals and across studies ${ }^{10,30-32}$ indicate that the airways are not simply passive recipients of inhaled microbiota and provide strong evidence for host selection. These microbial communities are present in infants ${ }^{30}$, and airways seem likely to be colonized in a way similar to other body surfaces ${ }^{33}$ during the weeks after birth.

In health, the oropharynx, the thoracic airways and the fluids from bronchoalveolar lavage (BAL; fluid washings obtained through a bronchoscope wedged into the subsegmental airways) contain similar microbiota $^{10,11}$, and the extent to which the lower airways are populated by aspiration from the upper respiratory tract is debated ${ }^{34}$. However, in disease, the presence and abundance of pathogens in the lung are not consistently reflected in the oropharynx ${ }^{10,34}$, which emphasizes that direct sampling of the lower airways and BAL are desirable in studies of the pulmonary microbiota ${ }^{34}$, with strict controls for contamination during bronchoscopy ${ }^{11}$ and laboratory analyses ${ }^{35}$. 


\section{Pulmonary infectious agents}

Pulmonary bacterial pathogens have been discovered almost entirely through microbial culture. Historically, the most important lung pathogen has been Mycobacterium tuberculosis. Between the 17th and 19th centuries, it accounted for one-fifth of all deaths in Europe and North America ${ }^{36}$. It may lie latent for years before causing a chronic cavitating lung infection that produces highly infective sputum (FIG. 1). After a marked decline in prevalence, due primarily to improvements in public health, M. tuberculosis infections are now increasing in incidence, with MDR strains spreading in many populations.

Another important pathogen is Streptococcus pneumoniae, the most frequently detected bacterial cause of acute pneumonia, which typically presents with high fever, lobar consolidation and pleurisy (FIG. 1). In addition, Haemophilus influenzae infection is common in patients with smoking-related lung disease, causing bronchial inflammation with or without patchy infiltration into the surrounding lung (bronchopneumonia). H. influenzae is underdetected by standard clinical culture methods ${ }^{37}$. Other Proteobacteria that less commonly cause acute lung infections include Moraxella spp. and Neisseria spp. Unencapsulated non-typeable $H$. influenzae (NTHI) strains are the main drivers of recurrent airway infection in COPD, together with Moraxella catarrhalis and S. pneumoniae ${ }^{38}$. Asthmatic airways appear to be similarly dominated by Proteobacteria (which may be Neisseria spp. rather than $H$. influenzae) and Firmicutes (potentially S. pneumoniae $)^{24-28}$, although asthmaassociated OTUs have yet to be defined by isolated culture.

The microorganisms described above have the ability to adhere to and invade other airway mucosal surfaces. In addition, S. pneumoniae and capsulated forms of $H$. influenzae and Neisseria meningitidis are the principal causes of bacterial meningitis, and S. pneumoniae, NTHI and $M$. catarrhalis also cause chronic infections of the middle ear (otitis media). All these organisms are commonly carried in the nose and oropharynx by healthy children (BOX 1), and extensive vaccination programmes are changing the prevalence of their capsulated forms. However, it is unencapsulated NTHI strains (which are difficult to vaccinate against) that drive recurrent airway infection in $\mathrm{COPD}^{38}$, and colonization with these strains is also associated with wheeze in infants ${ }^{24}$. In addition, unencapsulated strains of $S$. pneumoniae are carried by approximately $10 \%$ of children and cause otitis media ${ }^{39}$. Their role in respiratory disease has not been assessed. The carriage of S. pneumoniae and $H$. influenzae by a substantial proportion of healthy children indicates that colonization per se does not cause disease. The important factors that may cause the shift from colonization to invasion and overt disease (BOX 1) have not been extensively studied but may include concomitant viral infections, exposure to cigarette smoke and environmental pollution and progressive growth over the host microbiota ${ }^{40}$.

Interestingly, different bacterial pathogens are associated with the chronic infections that accompany cystic fibrosis and bronchiectasis. Pseudomonas aeruginosa, a facultative aerobic proteobacterium that causes opportunistic infection and is associated with antibiotic resistance, is an important pathogen, as are Burkholderia cepacia, Staphylococcus aureus and nontuberculous mycobacteria (BOX 1).

Notably, the microbiota of those with chronic lung diseases can contain abundant other potential pathogens; $H$. influenzae and S. pneumoniae most frequently dominate the sputum and airway microbiota of both patients with cystic fibrosis and patients with bronchiectasis. Common pathogens isolated by culture from patients with HAP and VAP include Pseudomonas spp. and other Gram-negative bacilli, staphylococci, streptococci and Haemophilus spp. ${ }^{41}$. Mixed or polymicrobial infections occur in patients who are immunocompromised or suffering from chronic lung infections, posing further therapeutic challenges ${ }^{42}$.

In addition to bacterial pulmonary disease, viral respiratory infections are common $^{43}$, and their ability to spread rapidly and cause epidemics has a major global impact. Although viral infections are not the subject of this review, it should be recognized that they do not act alone. $H$. influenzae was first discovered in 1892 during an influenza epidemic ${ }^{44}$, and bacterial pneumonia was the principal cause of death in the 1918-1919 Spanish flu pandemic ${ }^{45}$. Viral infections often trigger exacerbations of asthma and COPD, and multiple interactions can occur between viruses and common bacterial pathogens during acute infection $^{46}$. HIV infection, which supresses the adaptive immune system, increases the risk of pneumococcal pneumonia 20 -fold ${ }^{47}$. Mechanistically, influenza infection promotes adherence and invasion by S. pneumoniae ${ }^{48}$. In addition, interactions between rhinoviruses and $H$. influenzae augment rises in inflammatory markers and exacerbation severity in $\mathrm{COPD}^{49}$.

Finally, fungi form symbiotic as well as pathogenic relationships with bacterial and eukaryotic communities. Some fungal organisms can cause distinct syndromes of lung infection ${ }^{50}$. Furthermore, substantial fungal growths may be present in the microbiota of patients with cystic fibrosis and bronchiectasis ${ }^{51}$. Thus, important interactions seem likely between fungal and bacterial pathogens ${ }^{52}$. Invasive fungal disease associated with emergent organisms is increasing in incidence in immunocompromised hosts ${ }^{53}$. In addition, fungal pathogens may be important in HAP but have been incompletely studied ${ }^{18}$. The difficulty in isolating fungi by culture almost certainly means that detection rates do not reflect the prevalence of potentially pathogenic species in clinical specimens. Fungi can cause disease by varied mechanisms. Direct invasion of immunocompromised tissues may, at times, be less important than overexuberant host responses to the presence of the fungus. For example, in allergic bronchopulmonary aspergillosis, colonization of the airways by Aspergillus fumigatus is accompanied by elevated blood levels of immunoglobulin $\mathrm{E}$ and eosinophils. Treatment of the immune response with corticosteroids is currently given priority over fungal eradication.

\section{Current therapeutic strategies}

Although the aim of this article is to suggest improvements and new directions to the management of lung infections, it is worthwhile first to summarize contemporary diagnosis and treatment of these illnesses, with an emphasis on the problems that are faced.

Current diagnosis. Bacteriological diagnosis for lung infections in most clinical laboratories depends on isolating pathogens from patient samples by microbial culture. The technology for culture on agar plates was invented in 1880 in Berlin ${ }^{54}$. Plate culture can be effective in isolating pathogens, followed by testing for microbial sensitivities and identification by molecular techniques such as mass spectrometry $^{55}$. However, microbial culture has many subjective elements, beginning with pathogen differentiation from commensals by colony morphology. Failure to identify causal organisms occurs in 50\% of patients who have been hospitalized with pneumonia in intensive care units ${ }^{56}$, with 
similar low microbial diagnostic rates in pneumonia following immunosuppressive therapy or bone marrow transplants ${ }^{57,58}$. The administration of antibiotics before specimen collection is an omnipresent problem. Furthermore, the use of specialized culture media or anaerobic culture can stretch the capacity of routine clinical laboratories, and protocols may be erratically guided by clinical suspicion.

Deconvoluting the complex interactions between the microbiota and different pathogens in acute exacerbations or in the presence of polymicrobial infections is often beyond current diagnostic capabilities, which can make it difficult to make rational clinical decisions about antimicrobial therapy.

Current treatments. Although it is universally accepted that widespread use of antimicrobial agents is causing a global crisis in antibiotic resistance ${ }^{59}$, it has not been sufficiently recognized that antibiotic prescription for lung infections is a primary contributor to this problem ${ }^{6,8}$. In 2017, the WHO listed 12 antibiotic-resistant 'priority pathogens' that pose the greatest threat to human health; of these, four affect the lungs (P. aeruginosa, S. pneumoniae, $H$. influenzae and $S$. aureus).

Antibiotics for respiratory infections are usually given orally, with $\beta$-lactams often the drugs of first choice. Although the upper airway microbiota appears somewhat resilient and returns to the preantibiotic state quickly, in the gut, substantial selection for antibiotic-resistant organisms can be detected many months after treatment ${ }^{60}$. Because early treatment for lung infections

\section{Box 1 | The mobility and evolution of the lung microbiota}

Respiratory pathogens such as Streptococcus pneumoniae, Haemophilus influenzae, Neisseria spp. and Moraxella spp. are all commonly present in healthy individuals yet lead to disease in only some. They require human contact to spread, and the extent of the contact differs in many environments (see the figure). These bacteria may be carried in the nose and nasopharynx but cause disease in the lower airways and lung parenchyma. Factors that can trigger the switch between colonization and infection include the number of bacteria in vulnerable sites, synergism and nutrient competition with commensals ${ }^{122}$, intercurrent viral infections, cigarette smoking and air pollution. By contrast, bacteria that cause opportunistic infections of damaged lungs (for example, in patients with cystic fibrosis), such as Pseudomonas aeruginosa and nontuberculous mycobacteria, follow the exemplar of many enteropathogenic bacteria by having an environmental reservoir ${ }^{123}$ where they can persist before infecting susceptible hosts.

The potential for viruses to mutate and spread pandemically is universally feared. Less attention has been given to transmission of bacterial and fungal pathogens, although international travel, high population densities and selective pressures for pathogenicity provide fertile grounds for such events. For example, whole-genome sequencing of Mycobacterium tuberculosis isolates shows that this exclusively human pathogen emerged as a chronic infection 70,000 years ago, following modern humans out of Africa and evolving traits of virulence and transmissibility to take advantage of progressive human overcrowding ${ }^{124}$. M. tuberculosis sequencing can now trace where modern outbreaks arise and how they spread ${ }^{125}$.

The movement of pathogens within hospitals was first shown by whole-genome sequencing during an outbreak of lung infections with carbapenem-resistant Klebsiella pneumoniae ${ }^{126}$. Children with cystic fibrosis are highly prone to chronic lung infections and are thus subject to multiple antibiotic administrations, often within hospitals and their associated clinics. Patient-to-patient transmission of

virulent multidrug-resistant (MDR) Burkholderia cepacia was discovered in 1990 (REF. 127), before detection of transmission of MDR strains of P. aeruginosa ${ }^{128}$ and Mycobacterium abscessus ${ }^{129}$. Alarmingly, many M. abscessus infections are caused by dominant clones that have spread internationally ${ }^{130}$. Knowledge of these risks to patients with cystic fibrosis has led to aggressive measures to control transmission of MDR strains in hospitals and clinics ${ }^{131}$.

The spotlight has now turned to emerging fungal pathogens in cystic fibrosis ${ }^{132}$, exemplified by Scedosporium spp. ${ }^{133}$. In patients without cystic fibrosis hospitalized for a long time with lines or tubes entering their body, a recently emergent MDR Candida auris is

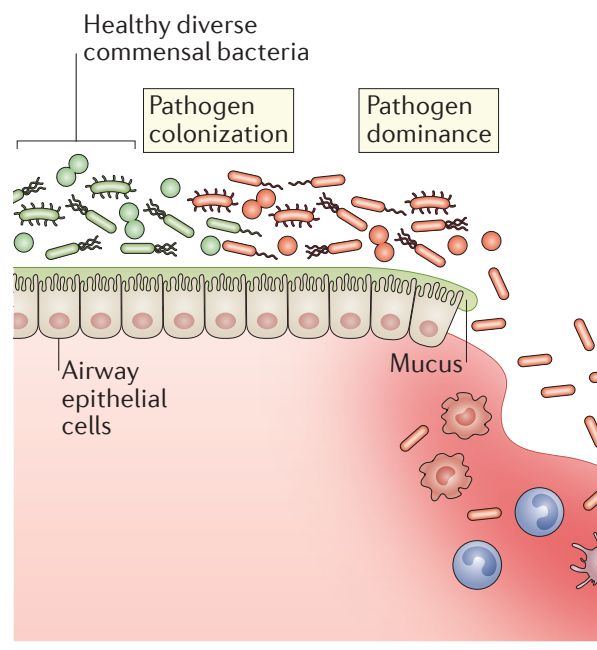

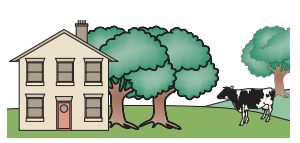

Rural populations

- Rich microbial

environment

- Small pathogen pool

- Low mixing

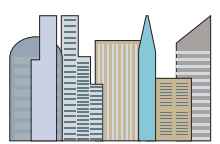

Cities

- Large pathogen pool • Naive immunity

- Much mixing

- Selection for AMR

- More virulent strains in circulation causing serious invasive infections with a mortality approaching $60 \%{ }^{134}$. C. auris infections were first reported in Japan, followed by reports from South Korea, India, Kuwait, South Africa, Pakistan and the United Kingdom and then South America and the United States ${ }^{134}$.

These studies show how cosmopolitan MDR strains spread nationally and internationally in a highly connected world, giving new meaning to the 'everything is everywhere' hypothesis ${ }^{135}$. The next decade promises to yield much more information about virulent strains of more mundane pathogens, such as $\mathrm{H}$. influenzae. It is almost certain that measures to control the spread of organisms from all clinical circumstances will need to be greatly changed. 
improves outcomes, most patients are given antibiotics without a microbial diagnosis, and British Thoracic Society guidelines urge empirical antibiotic use in patients treated for CAP in primary care ${ }^{61}$ (FIG. 2). In addition to patients' toxic and allergic responses to antibiotics, antibiotic damage to resident microbial populations considerably increases susceptibility to bowel infections ${ }^{62}$. Similar processes are possible in the lung, and the balance between the beneficial and detrimental effects of antibiotics could profitably be investigated in COPD, cystic fibrosis and bronchiectasis.

The therapeutic strategy in patients with pneumonia of unknown aetiology is to escalate the complexity and intensity of the antibiotic regimen, often with aminoglycosides, with the associated risks of selecting for and disseminating MDR (FIG. 2). The microbiological causes of HAP and VAP vary by location, and MDR to these organisms is almost universal. Broad-spectrum antibiotic therapy for $\mathrm{HAP}$ and VAP is given at the onset of disease ${ }^{41}$. De-escalation of antibiotics is recommended once culture data are available $e^{41}$, but this is unlikely to prevent selection for antimicrobial resistance (AMR) in the gut ${ }^{60}$.

Antibiotic prophylaxis is widely used in the management of bronchiectasis, as exemplified by children with cystic fibrosis ${ }^{63}$. The isolation of S. aureus and P. aeruginosa by microbial culture is related to worse outcomes, but antibiotic prophylaxis against staphylococci has no clear benefit and may increase carriage of $P$. aeruginos $a^{64}$. Attempting an early, targeted eradication of $P$. aeruginosa, similarly, has no definite beneficial effect on mortality or morbidity ${ }^{65}$.

\section{Evolving therapies}

Many of the mutations that confer AMR come with a fitness cost to the bacteria that carry them ${ }^{66}$, which offers the hope that finishing a course of antibiotics may eventually be followed by reversion of the microbiota towards its pre-antibiotic state. Continued efforts to prescribe fewer antibiotics are essential, but other innovative approaches to therapy are now possible. They are summarized in FICS 2,3 and discussed in more detail below.

Improved diagnostics. Modern sequencing technology offers a variety of alternative diagnostic approaches to traditional culture-based methods (BOX 2). An immediate benefit of sequence-based detection of the microbiota in clinical

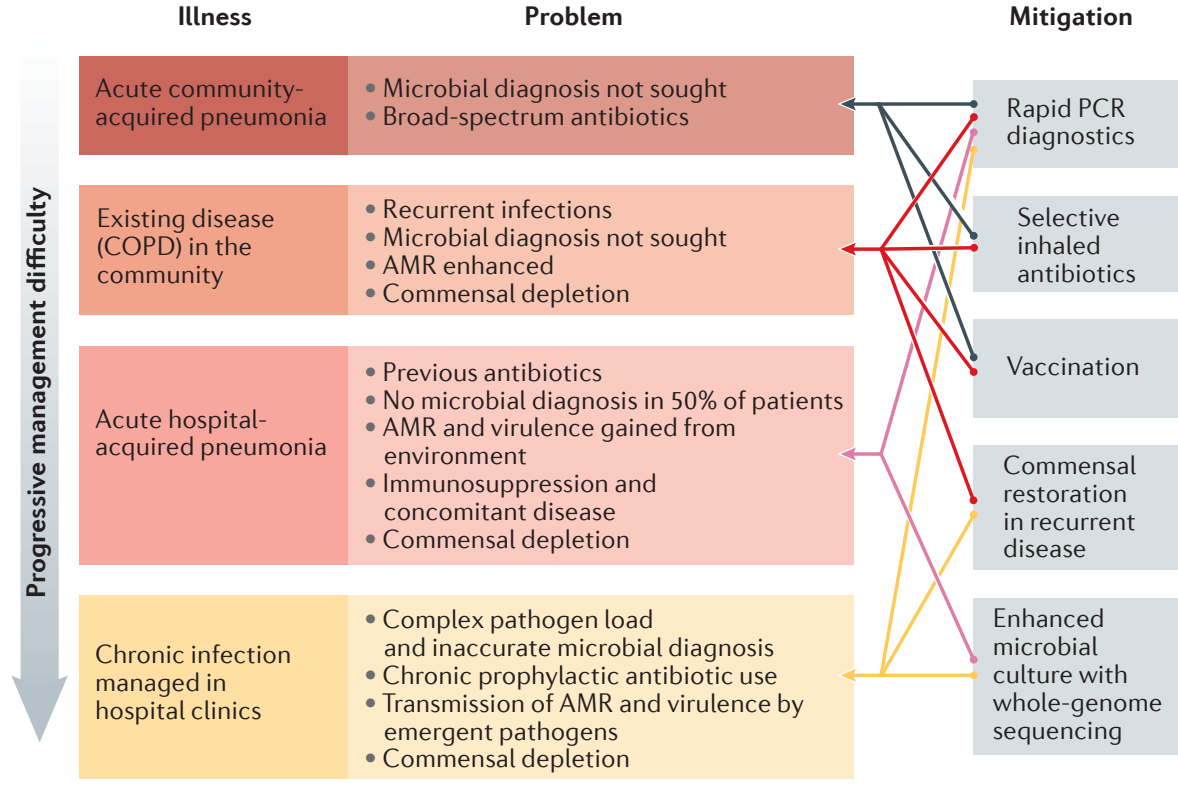

Figure 2 | Current therapeutic strategies for lung infections. The flow chart details problems with the current diagnosis and treatment strategies for various lung infections, from simple communityacquired pneumonia to the therapy of chronic bronchial infections. Potential generic improvements to disease management are shown on the right. AMR, antimicrobial resistance; COPD, chronic obstructive pulmonary disease.

samples is the prospect of improved accuracy of clinical diagnostics (FIG. 2). For example, 16S rRNA gene sequence analysis of DNA isolated from clinical specimens can better identify poorly described, rarely isolated or phenotypically aberrant strains. It can be routinely used for identification of mycobacteria (which are difficult to identify by culture-based methods) and can lead to the recognition of novel pathogens and bacteria that are resistant to standard clinical culture ${ }^{67-69}$ (BOX 2). By and large, $16 \mathrm{~S}$ rRNA gene sequencing from bacterial isolates has been envisaged for microbial identification $^{67-69}$. However, 16S rRNA gene analysis of the whole bacterial community from biological specimens such as sputum and samples collected from throat swabs may provide direct information of the presence of pathogens without the need for culture (BOX 2).

In contrast to open-ended sequence analysis of $16 \mathrm{~S}$ rRNA gene amplicons, specific quantitative PCR (qPCR) assays for common pathogens are already possible and may deliver results in a fraction of the time of standard clinical cultures. Additionally, qPCR with general primers can measure the overall number of all bacterial genomes, which can be combined with speciesspecific assays to interpret the relative dominance of a particular pathogen in the microbiota (as an organism accounting for $50 \%$ of the microbiota carries a different interpretation than one accounting for 2\%). Similar diagnostic possibilities exist for fungal pathogens by use of, for example, high-throughput sequencing of the amplified internal transcribed spacer 2 (ITS2) region ${ }^{70}$. Diagnostic fungal cultures are technically difficult and currently take 2-4 weeks of incubation ${ }^{71}$, so there are considerable possible speed and accuracy benefits in switching to sequence-based methods of detection ${ }^{72}$.

Problems with diagnosis by the $16 \mathrm{~S}$ rRNA gene and ITS2 sequencing derive from the accuracy of sequences in databases as well as those generated by the diagnostic laboratory and may result in an uncertain definition of species when based only on sequence information instead of on the traditional phenotypic classification of cultured microbial isolates ${ }^{67,68}$. In addition, multiple copies of the 16S rRNA gene may be present and show sequence variation in some bacterial species ${ }^{69}$. Limited $16 \mathrm{~S}$ rRNA gene sequence variation makes discrimination difficult between different Streptococcus spp., including S. pneumoniae, which may be overcome by sequencing other polymorphic loci ${ }^{73}$.

In the clinical context, rigorous control is necessary to prevent microbial nucleic acid contamination of swabs, containers of human fomites, PCR kits, sequencing kits and reagents ${ }^{35}$. Mixtures of bacteria that have a different resistance to lysis ${ }^{74}$ can be overcome 


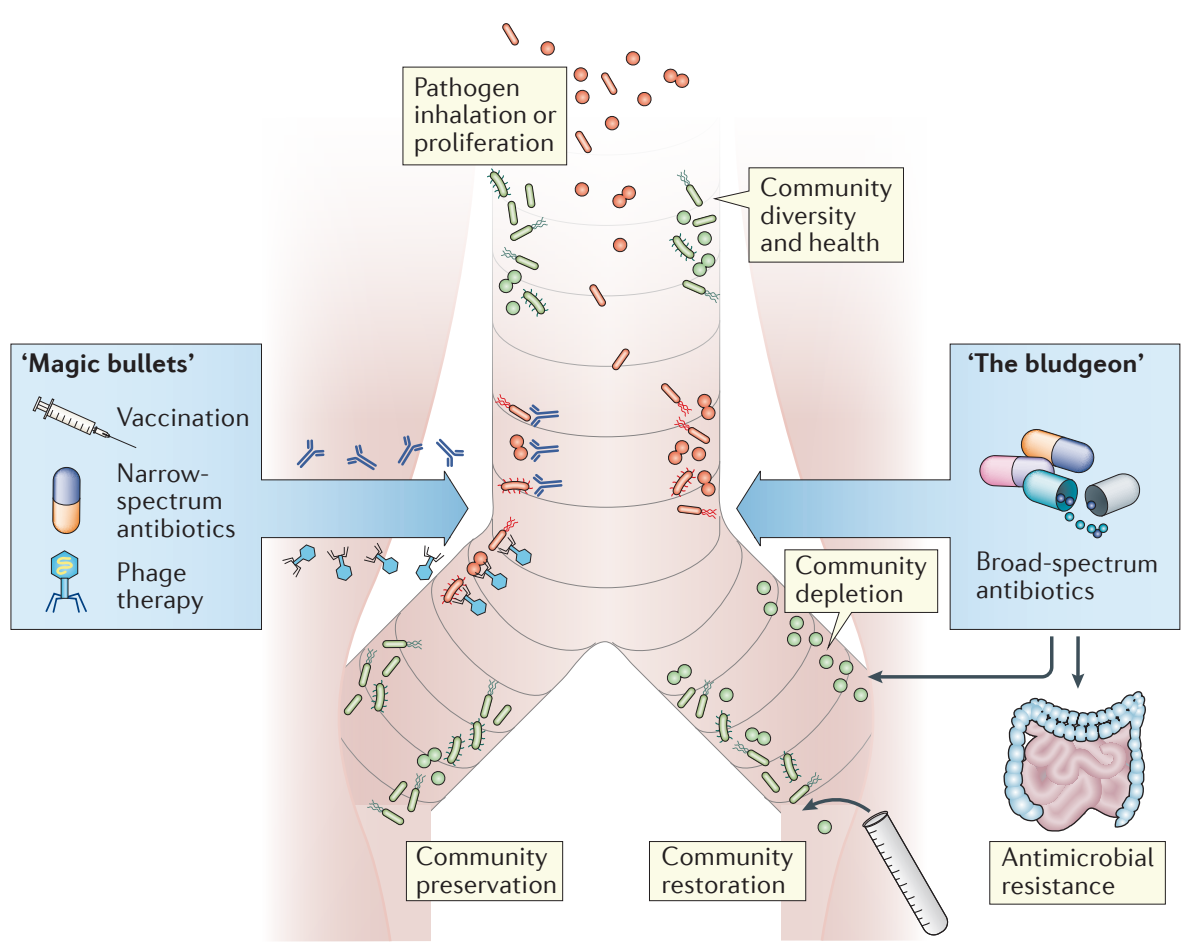

Figure 3 | New therapeutic strategies for lung infections. Healthy airways contain a diverse mixture of commensals. Lung infections with pathogens (red) are typically treated with systemic broadspectrum antibiotics (the bludgeon) that may deplete healthy commensals (green) and leave antibiotic-resistant organisms in the bowel as well as the lung. New therapeutic approaches (magic bullets) include vaccination, inhaled narrow-spectrum antibiotics with primary effects on particular pathogens and phage therapy. Commensal community restoration may replenish a depleted microbiota and improve resistance to subsequent reinfections.

by standardized methods, such as bead beating (high-energy mixing with grinding beads), with well-understood performances for important organisms. An important consideration is that DNA sequences will not distinguish between live and quiescent microorganisms or extracellular DNA from dead microorganisms.

Other than pathogens, most of the pulmonary microbiota have not been isolated or characterized in any detail ${ }^{29}$. Studies of the bowel microbiota have shown how improved culture followed by whole-genome sequencing can permit sophisticated analyses of human microbial communities in health and in disease $\mathrm{e}^{75}$. The diagnostic utility of the sequencing of strain-specific loci or of metagenomes will depend on future systematic culture and genomic sequencing of all the lung microbiota. The perception that human indigenous bacteria are largely recalcitrant to culture is inaccurate ${ }^{75}$, and simple measures may improve isolation of organisms from the lung. Anaerobic culture, for example, has not routinely been used for pulmonary specimens, despite the high numbers of anaerobic OTUs detected by $16 \mathrm{~S}$ RNA gene sequencing. Mucins may actively support the presence of some commensals ${ }^{76}$, and the addition of mucins to culture media may provide essential nutrients for colony growth.

Whole-genome sequencing of bacterial isolates has numerous applications to the problem of antibiotic resistance, including the development of novel antibiotics and diagnostic tests, the stewardship of available antibiotics to prevent AMR and the elucidation of factors that generate and perpetuate resistance ${ }^{77,78}$. Proof-of-principle studies for the value of whole-genome sequencing have come from investigations of M. tuberculosis ${ }^{79,80}$ and P. aeruginosa infections ${ }^{81}$. This systematic approach is easily extended to other pathogens such as S. pneumoniae and Haemophilus spp. isolated from patients with lung infections. A publicly accessible culture collection for the lung microbiota will encourage the development of model systems to investigate interactions between hosts, pathogens and commensals with straightforward replication of important results.

Metagenomic shotgun sequencing of bowel samples has delivered a mass of information about microbial processes in health and disease. Sputum, lung biopsy samples and brushings, unlike stool samples, are problematic for microbial metagenomics because the ratio of human to microbial DNA may be higher than 99:1. Strategies to enrich for microbial DNA before making sequencing libraries are technologically possible ${ }^{82}$ but not yet effective in practice. Promisingly, next-generation sequencing of RNA from BAL samples has proved effective in diagnosing viral and bacterial infections ${ }^{83}$.

Improved antibiotic regimens. The emergence of AMR in pathogens shares many factors in common with the emergence of drug-resistant malignant cells during chemotherapy for cancer. The management of AMR may be improved by strategies that are similar to those used for cancer chemotherapy, where the quantification of resistance mechanisms has been followed by specific countertherapies ${ }^{84}$. This approach has been exemplified by the development of $\beta$-lactamase inhibitors to counter penicillin resistance.

Extraordinarily, the experimental and theoretical basis that underlies antibiotic dosage regimens is limited and the results contentious ${ }^{85}$. High-dose antimicrobial chemotherapy has long been considered the best means of controlling drug resistance, but recent studies have shown that this can encourage the appearance of resistant strains at the same time as it releases them from ecological competition ${ }^{86}$. The effectiveness of the common use of combinations of antibiotics has also been questioned, and it has been suggested that in many circumstances, such combinations will actually increase pathogen load ${ }^{87}$. These findings, and the rising tide of AMR, indicate a need for an experimental re-examination of antibiotic regimens in common clinical states. To help this process, elements important in antibiotic resistance are increasingly accessible through genomic sequencing of pathogen isolates and also from partial-genome metagenomic sequence data $^{88}$, which allow a better anticipation of the response to antibiotics. Microbial sequencing can also be used to make decisions about undesirable consequences of antibiotic treatment of (as yet unidentified) keystone commensal organisms as well as about AMR emergence.

Inhaled antibiotics. The administration of oral antibiotics can result in long-lasting adverse alteration of gut microbial 
communities $^{60}$, which suggests that benefits will follow from reducing or avoiding exposure of gut organisms to antimicrobials (FIG. 3). Inhalations have been used medically for two millennia, and a wide range of aerosolized medications are now in use or in development for many diseases ${ }^{89}$. The pulmonary delivery of antibiotics (often as powders) for lung infections has shown success, with reduced dosing required and reductions in side effects $^{90}$. Aerosolized (inhaled) antibiotics may be more effective than their systemic counterparts in treating pneumonia ${ }^{91}$. When used to treat persistent airway infection in cystic fibrosis, inhaled antibiotics appear more effective than oral or parenteral therapy ${ }^{92}$. It has been suggested that inhaled antibiotics do not promote AMR, and a meta-analysis of studies in cystic fibrosis did not observe significant development of antibiotic-resistant organisms ${ }^{90,91}$.

A limited range of antibiotics is currently marketed for inhalation and approved for use in cystic fibrosis by the FDA and the European Medicines Agency ${ }^{93}$. These include tobramycin, colistin and nebulized aztreonam $^{93}$. Although not approved for other diseases, injectable formulations of gentamicin, tobramycin, amikacin, ceftazidime and amphotericin are currently nebulized 'off-label' to manage non-cystic fibrosis bronchiectasis, drug-resistant nontuberculous mycobacterial infections, VAP and airway infections occurring after transplant ${ }^{93}$.

The advantages of inhaled therapy encourage a search, beyond the treatment of chronic infections in cystic fibrosis ${ }^{93}$, for novel antibiotics with chemical characteristics that encourage retention in the lung (FIG. 3). A more selective antibiotic specificity (narrow spectrum) towards major pathogens such as $H$. influenzae is also desirable (FIG. 2). Patients with COPD who suffer frequent lower respiratory tract infections, which contribute enormously to AMR in the community ${ }^{8}$, would be an ideal group for the comparison of inhaled and systemic antibiotic therapies.

\section{Phage therapy. In 1917, bacteriophages} were discovered to cause epizootic infections of bacteria and were used for antibacterial therapy and prophylaxis ${ }^{94}$. However, a poor understanding of their biology and the advent of antibiotics curtailed research after 1945 (REF. 94). The early literature suggests that phage therapy is very effective ${ }^{94}$, and there has been a modern renewal of interest in response to

\section{Box 2 | Complex clinical uses of microbial community analyses}

DNA and RNA sequence analysis of specimens from the lung may be of direct clinical benefit to the management of lung infections. However, there are obstacles yet to be overcome.

- Simple PCR assays can be used to quantify known pathogens. However, not enough is yet known about many of the unencapsulated Proteobacteria, including non-typeable Haemophilus influenzae and Neisseria spp. that dominate in chronic obstructive pulmonary disease and asthma. The systematic isolation and sequencing of these organisms is desirable for the successful development of rapid diagnostic assays.

- In contrast to culture techniques, a simple presence or absence of a particular bacterial operational taxonomic unit may not be sufficient to guide therapy. Absolute counts (by quantitative PCR or digital PCR) will be more helpful. Criteria are needed to differentiate between asymptomatic carriage of common pathogens and the presence of disease. Concomitant measure of the total bacterial load can be used to estimate the degree of pathogen dominance.

- Sequence analysis also allows consideration of synergistic effects between members of a microbial community, avoiding the misleading assumption of 'one pathogen, one disease' (REF. 136).

- It will be desirable to develop measures of the health of a patient's microbiome, identify optimal patterns of diversity and resilience and establish the presence or absence of keystone organisms associated with health. These parameters will permit public health promotion of infection resistance in otherwise susceptible individuals and environments.

- The effects of antibiotics will be assessed by their impact on whole microbial communities so that antimicrobial actions may be balanced between commensals and pathogens.

- Mixed infections are common, particularly in the presence of underlying lung disease. The identification of all pathogens in an infection and a knowledge of pathogen synergies will improve treatments.

- Metagenomic transcriptomic analysis (RNA) of bacterial gene expression may identify targets for therapy in particular infections. Metagenomic analysis (DNA) of lung samples is currently severely hampered by high concentrations of human DNA. Single-cell transcriptomics of bacteria purified from samples may be helpful.

- Antibiotic sensitivities can currently be predicted by sequence analysis of bacterial isolates. The application of this technique to complex microbial mixtures may give a measure of general community resistance. Pathogen-specific signatures may be discerned by unique sequences within the community. Alternatively, single-cell sequencing may identify the antimicrobial resistance profile of individual organisms.

- Epidemiological surveys of the transmission and community carriage of common respiratory pathogens, such as non-typeable $H$. influenzae, will underpin strategies to reduce transmission and prevent colonization.

increasing $\mathrm{AMR}^{95}$. Phages can be modified genetically to induce desirable biological and therapeutic properties ${ }^{96}$. Importantly, phage infection leads to only transient changes in the microbial communities that harbour targeted pathogens ${ }^{97}$. Despite these advantages, phage therapy is not proving easy to develop within the stringent regulatory requirements for new treatments ${ }^{8}$. Nevertheless, it has shown encouraging results for the treatment of $P$. aeruginosa infections in patients with cystic fibrosis ${ }^{99,100}$ and has major potential for targeted treatment of more common pathogens, such as H. influenzae (FIG. 3).

\section{Restoring bacterial communities.}

Manipulation of the microbiota confers colonization resistance to intestinal infections ${ }^{101-103}$, and faecal microbiota transplantation is markedly effective in treating the severe illnesses associated with persistent Clostridium difficile infection ${ }^{104}$ and inflammatory bowel disease $\mathrm{e}^{105}$. There is good reason to consider similar possibilities in the lungs (FIG. 3).

Disordered microbial communities that have a loss of commensal diversity are a feature of asthma ${ }^{10,106}$, which may be a reasonable pulmonary target for interventional trials. Community restoration might also be of benefit after long-term antibiotic use in children with cystic fibrosis or primary ciliary dyskinesia or in adults with COPD. However, in contrast to faecal microbiota transplantation, healthy individuals do not spontaneously provide airway microbiota in a form suitable for transplantation; in addition, little is known about the characteristics of lower-airway commensals, and pulmonary pathogens commonly colonize the airways without causing disease.

A structured approach to identifying bowel protective organisms has been exemplified for C. difficile infections ${ }^{102}$. 
Similar strategies for the airway microbiota may identify the minimum number of commensal organisms that might provide protection against colonization with pathogenic Proteobacteria and Streptococcus spp. or could be used to replace a microbial community with particularly high levels of AMR. The organisms to be used may vary for different diseases and different individuals. The dose of bacteria as well as the species to be introduced is an important consideration. In an analogy of restoring a garden, therapy with antimicrobials to remove pathogens (weeds) before treatment may be helpful. As with other novel therapies, an ethical framework will need to be established around early-stage clinical trials to examine safety before therapies can be explored.

Vaccination. The potential for vaccination to have an impact on common lung infections (FIG. 3) is exemplified by invasive infections with S. pneumoniae ${ }^{107}$. Although vaccination in the first years of life does not confer long-lasting immunity ${ }^{108}$, prevention of invasive disease by $S$. pneumoniae with AMR is achievable ${ }^{109}$. The rate of antibiotic-resistant, invasive pneumococcal infections has decreased in young children and older adults with the widespread use of pneumococcal conjugate vaccines ${ }^{110}$, and vaccination is effective in preventing pneumonia in children and adults following splenectomy ${ }^{111}$.

Respiratory infections caused by NTHI result in enormous global morbidity ${ }^{38}$. Effective $H$. influenzae vaccines have been directed against capsular antigens and are given routinely in childhood for meningitis and otitis media in most Western societies. However, the unencapsulated NTHI strains that commonly cause lung diseases are not influenced by current vaccines ${ }^{112-114}$. A vaccine against NTHI would prevent disease and reduce antibiotic use $^{8}$, most clearly in the context of COPD exacerbations ${ }^{115,116}$ but also in CAP, cystic fibrosis and chronic bronchitis. An ideal vaccine antigen should be conserved among strains, have abundant epitopes on the bacterial surface and induce protective immune responses ${ }^{8}$. A conjugate vaccine against protein $\mathrm{D}$ (a highly conserved and stable protein in the outer membrane of encapsulated and unencapsulated H. influenzae strains), although only partially effective, has demonstrated the feasibility of vaccination for $\mathrm{NTHI}^{8,114,117}$.

Characterization of the asthmatic airway microbiome is at an early stage, but there is a consensus that there is an overgrowth of Neisseria spp. and Moraxella spp. as well as of Haemophilus spp. and Streptococcus spp. ${ }^{10,28,30,118,119}$. In addition, $S$. pneumoniae has a high reported carriage rate $(45 \%)$ in children with asthma ${ }^{108}$. Should these bacteria be shown definitively to be causing airway inflammation, the success of vaccination against such organisms leads to the intriguing possibility of eventual vaccination to protect against childhood asthma. Inherent in a vaccination strategy would be the recognition that many of the organisms associated with asthma and COPD are likely to be unencapsulated strains. This further emphasizes the need for a concerted attempt to isolate and sequence the lower-airway microbiota.

Vaccines against $P$. aeruginosa are not effective in preventing infections in patients with cystic fibrosis ${ }^{120}$, perhaps suggesting that vaccinations have less value in patients with infections that flourish in luminal secretions, which may be at a distance from mucosal defence mechanisms.

A better understanding of the population distributions of potentially pathogenic Proteobacteria as well as H. influenzae and $S$. pneumoniae in the healthy population, as well as in patients with asthma and patients with COPD, is important to help develop rational vaccination strategies. Effective vaccination is likely to be followed by the evolution of population serotypes, which would impart a need for continuous vaccine development ${ }^{121}$. However, the global numbers of children and adults at risk suggest that the effort would be worthwhile.

\section{Conclusions}

Lung infections have enormous effects on human health, but current treatments are imperfect and fuel a global engine for AMR. Genome sequencing is fundamentally changing our understanding of the airway microbiome in health and disease, not least by revealing the profound pressures that drive the evolution and spread of pathogenic microorganisms. Genomic studies of opportunistic pathogens that accompany cystic fibrosis show how easily hyper-resistant and virulent clones can disseminate from clinic to clinic across the world. These studies provide an exemplar for investigation of the epidemiological suspicion that the evolution and transmission of apparently mundane pathogens may be common. Sequence-based techniques are ready to transform the microbial diagnosis of common lung infections and are the first priority for improving management of and minimizing AMR. A systematic understanding of the healthy pulmonary microbiota is a necessary platform from which to build new therapies that may include novel inhaled antibiotics, phages, vaccination and techniques to restore healthy microbial communities.

William O. C. M. Cookson, Michael J. Cox and Miriam F. Moffatt are at the Asmarley Centre for Genomic Medicine, National Heart and Lung Institute, Imperial College London, Dovehouse Street, London SW3 6LY, UK.

Correspondence to W.O.C.M.C. w.cookson@imperial.ac.uk

doi: $10.1038 /$ nrmicro.2017.122 Published online 24 Oct 2017

1. Adams, W. C. California Air Resources Board contract no. A033-205: Measurement of breathing rate and volume in routinely performed daily activities. California Air Resources Board https://www.arb.ca.gov/ research/apr/past/a033-205.pdf (1993).

2. Weibel, E. R. \& Gomez, D. M. Architecture of the human lung. Use of quantitative methods establishes fundamental relations between size and number of lung structures. Science 137, 577-585 (1962).

3. Hasleton, P. S. The internal surface area of the adult human lung. J. Anat. 112, 391-400 (1972).

4. Bowers, R. M. et al. Sources of bacteria in outdoor air across cities in the midwestern United States. Appl. Environ. Microbiol. 77, 6350-6356 (2011).

5. Helander, H. F. \& Fandriks, L. Surface area of the digestive tract - revisited. Scand. J. Gastroenterol. 49, 681-689 (2014)

6. Guest, J. F. \& Morris, A. Community-acquired pneumonia: the annual cost to the National Health Service in the UK. Eur. Respir. J. 10, 1530-1534 (1997).

7. Adriaenssens, N. et al. European Surveillance of Antimicrobial Consumption (ESAC): outpatient antibiotic use in Europe. J. Antimicrob. Chemother. 66 (Suppl 6.), vi3-vi12 (2011).

8. Murphy, T. F. Vaccines for nontypeable Haemophilus influenzae: the future is now. Clin. Vaccine Immunol. 22, 459-466 (2015).

9. Dickson, R. P., Erb-Downward, J. R. \& Huffnagle, G. B. The role of the bacterial microbiome in lung disease. Expert Rev. Respir. Med. 7, 245-257 (2013).

10. Hilty, M. et al. Disordered microbial communities in asthmatic airways. PLoS ONE 5, e8578 (2010).

11. Charlson, E. S. et al. Topographical continuity of bacterial populations in the healthy human respiratory tract. Am. J. Respir. Crit. Care Med. 184, 957-963 (2011).

12. Brook, I. Bacterial interference. Crit. Rev. Microbiol. 25, 155-172 (1999)

13. Reid, G., Howard, J. \& Gan, B. S. Can bacterial interference prevent infection? Trends Microbiol. 9, 424-428 (2001).

14. Falagas, M. E., Rafailidis, P. I. \& Makris, G. C. Bacterial interference for the prevention and treatment of infections. Int. J. Antimicrob. Agents 31, 518-522 (2008).

15. Pamer, E. G. Resurrecting the intestinal microbiota to combat antibiotic-resistant pathogens. Science 352 535-538 (2016)

16. Ferkol, T. $\&$ Schraufnagel, D. The global burden of respiratory disease. Ann. Am. Thorac Soc. 11, 404-406 (2014)

17. Walker, C. L. et al. Global burden of childhood pneumonia and diarrhoea. Lancet 381, 1405-1416 (2013).

18. Park, D. R. The microbiology of ventilator-associated pneumonia. Respir. Care 50, 742-765 (2005).

19. Goss, C. H. \& Burns, J. L. Exacerbations in cystic fibrosis. 1: Epidemiology and pathogenesis. Thorax 62, 360-367 (2007)

20. Donaldson, G. C., Seemungal, T. A., Bhowmik, A. \& Wedzicha, J. A. Relationship between exacerbation frequency and lung function decline in chronic obstructive pulmonary disease. Thorax $\mathbf{5 7}, 847-852$ (2002). 
21. Beasley, R. \& The International Study of Asthma and Allergies in Childhood (ISAAC) Steering Committee. Worldwide variation in prevalence of symptoms of asthma, allergic rhinoconjunctivitis, and atopic eczema: ISAAC. Lancet 351, 1225-1232 (1998)

22. Cookson, W. The immunogenetics of asthma and eczema: a new focus on the epithelium. Nat. Rev. Immunol. 4, 978-988 (2004).

23. Johnston, S. et al. Community study of role of viral infections in exacerbations of asthma in 9-11 year old children. BMJ 310, 1225-1229 (1995).

24. Bisgaard, H. et al. Childhood asthma after bacterial colonization of the airway in neonates. N. Engl. J. Med. 357, 1487-1495 (2007)

25. Green, B. J. et al. Potentially pathogenic airway bacteria and neutrophilic inflammation in treatment resistant severe asthma. PLOS ONE 9, e 100645 (2014).

26. Huang, Y. J. et al. Airway microbiota and bronchial hyperresponsiveness in patients with suboptimally controlled asthma. J. Allergy Clin. Immunol. 127 372-381.e3 (2011).

27. Goleva, E. et al. The effects of airway microbiome on corticosteroid responsiveness in asthma. Am. J. Respir. Crit. Care Med. 188, 1193-1201 (2013).

28. Huang, Y. J. \& Boushey, H. A. The microbiome in asthma. J. Allergy Clin. Immunol. 135, 25-30 (2015).

29. Man, W. H., de Steenhuijsen Piters, W. A. \& Bogaert, D. The microbiota of the respiratory tract: gatekeeper to respiratory health. Nat. Rev. Microbiol. 15, 259-270 (2017)

30. Cardenas, P. A. et al. Upper airways microbiota in antibiotic-naive wheezing and healthy infants from the tropics of rural Ecuador. PLOS ONE 7, e46803 (2012).

31. Stearns, J. C. et al. Culture and molecular-based profiles show shifts in bacterial communities of the upper respiratory tract that occur with age. ISME J. 9 1246-1259 (2015).

32. Molyneaux, P. L. et al. The role of bacteria in the pathogenesis and progression of idiopathic pulmonary fibrosis. Am. J. Respir. Crit. Care Med. 190, 906-913 (2014).

33. Twigg, H. L. et al. Use of bronchoalveolar lavage to assess the respiratory microbiome: signal in the noise Lancet Respir. Med. 1, 354-356 (2013).

34. Dickson, R. P. et al. Spatial variation in the healthy human lung microbiome and the adapted island model of lung biogeography. Ann. Am. Thorac Soc. 12 821-830 (2015)

35. Salter, S. J. et al. Reagent and laboratory contamination can critically impact sequence-based microbiome analyses. BMC Biol. 12, 87 (2014)

36. Wilson, L. G. Commentary: Medicine, population, and tuberculosis. Int. J. Epidemiol. 34, 521-524 (2005).

37. Kennedy, W. A. et al. Incidence of bacterial meningitis in Asia using enhanced CSF testing: polymerase chain reaction, latex agglutination and culture. Epidemiol. Infect. 135, 1217-1226 (2007)

38. Sethi, S. \& Murphy, T. F. Infection in the pathogenesis and course of chronic obstructive pulmonary disease. N. Engl. J. Med. 359, 2355-2365 (2008)

39. Keller, L. E., Robinson, D. A. \& McDaniel, L. S. Nonencapsulated Streptococcus pneumoniae: emergence and pathogenesis. mBio 7, e01792 (2016).

40. Siegel, S. J. \& Weiser, J. N. Mechanisms of bacterial colonization of the respiratory tract. Annu. Rev. Microbiol. 69, 425-444 (2015)

41. American Thoracic Society $\&$ Infectious Diseases Society of America. Guidelines for the management of adults with hospital-acquired, ventilator-associated, and healthcare-associated pneumonia. Am. J. Respir. Crit. Care Med. 171, 388-416 (2005).

42. Holcombe, L. J., O'Gara, F. \& Morrissey, J. P. Implications of interspecies signaling for virulence of bacterial and fungal pathogens. Future Microbiol. 6 , 799-817 (2011).

43. Lessler, J. et al. Incubation periods of acute respiratory viral infections: a systematic review. Lancet Infect. Dis. 9, 291-300 (2009).

44. Kuhnert, P. \& Christensen, H. Pasteurellaceae Biology, Genomics and Molecular Aspects (Caister Academic Press, 2008)

45. Morens, D. M., Taubenberger, J. K. \& Fauci, A. S. Predominant role of bacterial pneumonia as a cause of death in pandemic influenza: implications for pandemic influenza preparedness. J. Infect. Dis. 198 , 962-970 (2008)

46. Bosch, A. A., Biesbroek, G., Trzcinski, K. Sanders, E. A. \& Bogaert, D. Viral and bacterial interactions in the upper respiratory tract. PLoS Pathog. 9, e1003057 (2013).
47. Scott, J. A. et al. Aetiology, outcome, and risk factors for mortality among adults with acute pneumonia in Kenya. Lancet 355, 1225-1230 (2000)

48. McCullers, J. A. Insights into the interaction between influenza virus and pneumococcus. Clin. Microbiol. Rev. 19, 571-582 (2006).

49. Wilkinson, T. M. et al. Effect of interactions between lower airway bacterial and rhinoviral infection in exacerbations of COPD. Chest 129, 317-324 (2006)

50. Wheat, L. J., Goldman, M. \& Sarosi, G. State-of-the-art review of pulmonary fungal infections. Semin. Respir. Infect. 17, 158-181 (2002).

51. Pihet, M. et al. Occurrence and relevance of filamentous fungi in respiratory secretions of patients with cystic fibrosis - a review. Med. Mycol. 47 387-397 (2009)

52. Kastman, E. K. et al. Biotic interactions shape the ecological distributions of Staphylococcus species. mBio 7, e01157-16 (2016).

53. Enoch, D. A., Ludlam, H. A. \& Brown, N. M. Invasive fungal infections: a review of epidemiology and management options. J. Med. Microbiol. 55, 809-818 (2006).

54. Hesse, W. Walther and Angelina Hesse - early contributors to bacteriology. ASM News 58, 425-428 (1992)

55. Croxatto, A., Prod'hom, G. \& Greub, G. Applications of MALDI-TOF mass spectrometry in clinical diagnostic microbiology. FEMS Microbiol. Rev. 36, 380-407 (2012)

56. Waters, B. \& Muscedere, J. A. 2015 update on ventilator-associated pneumonia: new insights on its prevention, diagnosis, and treatment. Curr. Infect. Dis. Rep. 17, 496 (2015)

57. Joos, L. et al. Pulmonary infections diagnosed by BAL: a 12-year experience in 1066 immunocompromised patients. Respir. Med. 101, 93-97 (2007)

58. Boyton, R. J. Infectious lung complications in patients with HIV/AIDS. Curr. Opin. Pulm. Med. 11, 203-207 (2005).

59. Bush, K. et al. Tackling antibiotic resistance. Nat. Rev. Microbiol. 9, 894-896 (2011).

60. Zaura, E. et al. Same exposure but two radically different responses to antibiotics: resilience of the salivary microbiome versus long-term microbial shifts in feces. mBio 6, e01693-15 (2015).

61. Lim, W. S. et al. BTS guidelines for the management of community acquired pneumonia in adults: update 2009. Thorax 64 (Suppl. 3), iii 1-iii55 (2009).

62. Freter, R. In vivo and in vitro antagonism of intestina bacteria against Shigella flexneri: II. The inhibitory mechanism. J. Infect. Dis. 110, 38-46 (1962).

63. Mogayzel, P. J. Jr. et al. Cystic Fibrosis Foundation pulmonary guideline. Pharmacologic approaches to prevention and eradication of initial Pseudomonas aeruginosa infection. Ann. Am. Thorac Soc. 11, $1640-1650$ (2014)

64. Smyth, A. R. \& Walters, S. Prophylactic antistaphylococcal antibiotics for cystic fibrosis. Cochrane Database Syst. Rev. 11, CD001912 (2014).

65. Langton Hewer, S. C. \& Smyth, A. R. Antibiotic strategies for eradicating Pseudomonas aeruginosa in people with cystic fibrosis. Cochrane Database Syst. Rev. 11, CD004197 (2014).

66. Melnyk, A. H., Wong, A. \& Kassen, R. The fitness costs of antibiotic resistance mutations. Evol. Appl. 8, 273-283 (2015).

67. Clarridge, J. E. III. Impact of 16S rRNA gene sequence analysis for identification of bacteria on clinical microbiology and infectious diseases. Clin. Microbiol. Rev. 17, 840-862 (2004)

68. Woo, P. C., Lau, S. K., Teng, J. L., Tse, H. \& Yuen, K. Y Then and now: use of 16S rDNA gene sequencing for bacterial identification and discovery of novel bacteria in clinical microbiology laboratories. Clin. Microbiol. Infect. 14, 908-934 (2008).

69. Janda, J. M. \& Abbott, S. L. 16S rRNA gene sequencing for bacterial identification in the diagnostic laboratory: pluses, perils, and pitfalls. J. Clin. Microbiol. 45, 2761-2764 (2007).

70. Koljalg, U. et al. Towards a unified paradigm for sequence-based identification of fungi. Mol. Ecol. 22 5271-5277 (2013)

71. Bosshard, P. P. Incubation of fungal cultures: how long is long enough? Mycoses 54, e539-e545 (2011).

72. Balajee, S. A. et al. Sequence-based identification of Aspergillus, Fusarium, and Mucorales species in the clinical mycology laboratory: where are we and where should we go from here? J. Clin. Microbiol. 47. 877-884 (2009).
73. Hanage, W. P. et al. Using multilocus sequence data to define the pneumococcus. J. Bacteriol. 187 6223-6230 (2005).

74. Vebø, H. C., Karlsson, M. K., Avershina, E., Finnby, L. \& Rudi, K. Bead-beating artefacts in the Bacteroidetes to Firmicutes ratio of the human stool metagenome. J. Microbiol. Methods 129, 78-80 (2016).

75. Browne, H. P. et al. Culturing of 'unculturable' human microbiota reveals novel taxa and extensive sporulation. Nature 533, 543-546 (2016).

76. Linden, S. K., Sutton, P., Karlsson, N. G., Korolik, V. \& McGuckin, M. A. Mucins in the mucosal barrier to infection. Mucosal Immunol. 1, 183-197 (2008).

77. Koser, C. U., Ellington, M. J. \& Peacock, S. J. Whole-genome sequencing to control antimicrobial resistance. Trends Genet. 30, 401-407 (2014).

78. Punina, N. V., Makridakis, N. M., Remnev, M. A. \& Topunov, A. F. Whole-genome sequencing targets drug-resistant bacterial infections. Hum. Genom. 9, 19 (2015).

79. Walker, T. M. et al. Whole-genome sequencing for prediction of Mycobacterium tuberculosis drug susceptibility and resistance: a retrospective cohort study. Lancet Infect. Dis. 15, 1193-1202 (2015).

80. Bradley, P. et al. Rapid antibiotic-resistance predictions from genome sequence data for Staphylococcus aureus and Mycobacterium tuberculosis. Nat. Commun. 6, 10063 (2015).

81. Winstanley, C. et al. Newly introduced genomic prophage islands are critical determinants of in vivo competitiveness in the Liverpool Epidemic Strain of Pseudomonas aeruginosa. Genome Res. 19, 12-23 (2009).

82. Feehery, G. R. et al. A method for selectively enriching microbial DNA from contaminating vertebrate host DNA. PLOS ONE 8, e76096 (2013).

83. Fischer, N. et al. Evaluation of unbiased next-generation sequencing of RNA (RNA-seq) as a diagnostic method in influenza virus-positive respiratory samples. J. Clin. Microbiol. 53, 2238-2250 (2015).

84. Coley, H. M. Mechanisms and strategies to overcome chemotherapy resistance in metastatic breast cancer. Cancer Treat. Rev. 34, 378-390 (2008).

85. Kupferschmidt, K. Resistance fighters. Science 352 758-761 (2016)

86. Day, T. \& Read, A. F. Does high-dose antimicrobial chemotherapy prevent the evolution of resistance? PLoS Comput. Biol. 12, e 1004689 (2016).

87. Pena-Miller, $\mathrm{R}$, et al. When the most potent combination of antibiotics selects for the greatest bacterial load: the smile-frown transition. PLOS Biol. 11, e 1001540 (2013).

88. McArthur, A. G. et al. The comprehensive antibiotic resistance database. Antimicrob. Agents Chemother. 57, 3348-3357 (2013)

89. Rubin, B. K. Pediatric aerosol therapy: new devices and new drugs. Respir. Care 56, 1411-1423 (2011).

90. Traini, D. \& Young, P. M. Delivery of antibiotics to the respiratory tract: an update. Expert Opin. Drug Deliv. 6, 897-905 (2009)

91. Palmer, L. B. ¿ Smaldone, G. C. Reduction of bacterial resistance with inhaled antibiotics in the intensive care unit. Am. J. Respir. Crit. Care Med. 189, 1225-1233 (2014)

92. Ryan, G., Singh, M. \& Dwan, K. Inhaled antibiotics for long-term therapy in cystic fibrosis. Cochrane Database Syst. Rev. 3, CD001021 (2011).

93. Quon, B. S., Goss, C. H. \& Ramsey, B. W. Inhaled antibiotics for lower airway infections. Ann. Am. Thorac Soc. 11, 425-434 (2014).

94. Summers, W. C. Bacteriophage therapy. Annu. Rev. Microbiol. 55, 437-451 (2001).

95. Young, R. \& Gill, J. J. Phage therapy redux - what is to be done? Science 350, 1163-1164 (2015).

96. Nobrega, F. L., Costa, A. R., Kluskens, L. D. \& Azeredo, J. Revisiting phage therapy: new applications for old resources. Trends Microbiol. 23, 185-191 (2015).

97. Reyes, A., Wu, M., McNulty, N. P., Rohwer, F. L. \& Gordon, J. I. Gnotobiotic mouse model of phagebacterial host dynamics in the human gut. Proc. Natl Acad. Sci. USA 110, 20236-20241 (2013).

98. Servick, K. Beleaguered phage therapy trial presses on. Science 352, 1506 (2016)

99. Alemayehu, D. et al. Bacteriophages phiMR299-2 and phiNH-4 can eliminate Pseudomonas aeruginosa in the murine lung and on cystic fibrosis lung airway cells. mBio 3, e00029-12 (2012). 
100. Debarbieux, L. et al. Bacteriophages can treat and prevent Pseudomonas aeruginosa lung infections. J. Infect. Dis. 201, 1096-1104 (2010).

101. He, X., McLean, J. S., Guo, L., Lux, R. \& Shi, W. The social structure of microbial community involved in colonization resistance. ISME J. 8, 564-574 (2014).

102. Lawley, T. D. et al. Targeted restoration of the intestinal microbiota with a simple, defined bacteriotherapy resolves relapsing Clostridium difficile disease in mice. PLoS Pathog. 8, e 1002995 (2012).

103. Adamu, B. O. \& Lawley, T. D. Bacteriotherapy for the treatment of intestinal dysbiosis caused by Clostridium difficile infection. Curr. Opin. Microbiol. 16, 596-601 (2013).

104. Gough, E., Shaikh, H. \& Manges, A. R. Systematic review of intestinal microbiota transplantation (fecal bacteriotherapy) for recurrent Clostridium difficile infection. Clin. Infect. Dis. 53, 994-1002 (2011).

105. Colman, R. J. \& Rubin, D. T. Fecal microbiota transplantation as therapy for inflammatory bowel disease: a systematic review and meta-analysis. J. Crohns Colitis 8, 1569-1581 (2014).

106. Dickson, R. P., Martinez, F. J. \& Huffnagle, G. B. The role of the microbiome in exacerbations of chronic lung diseases. Lancet 384, 691-702 (2014).

107. Conklin, L. et al. Systematic review of the effect of pneumococcal conjugate vaccine dosing schedules on vaccine-type invasive pneumococcal disease among young children. Pediatr. Infect. Dis. J. 33 (Suppl. 2), S109-S118 (2014).

108. Esposito, S. et al. Streptococcus pneumonia colonisation in children and adolescents with asthma: impact of the heptavalent pneumococcal conjugate vaccine and evaluation of potential effect of thirteenvalent pneumococcal conjugate vaccine. BMC Infect. Dis. 16, 12 (2016)

109. Hampton, L. M. et al. Prevention of antibioticnonsusceptible Streptococcus pneumoniae with conjugate vaccines. J. Infect. Dis. 205, 401-411 (2012).

110. Kyaw, M. H et al. Effect of introduction of the pneumococcal conjugate vaccine on drug-resistant Streptococcus pneumoniae. N. Engl. J. Med. 354 1455-1463 (2006).

111. Ammann, A. J. et al. Polyvalent pneumococcalpolysaccharide immunization of patients with sicklecell anemia and patients with splenectomy. N. Engl. J. Med. 297, 897-900 (1977)

112. Bou, R. et al. Prevalence of Haemophilus influenzae pharyngeal carriers in the school population of Catalonia. Eur. J. Epidemiol. 16, 521-526 (2000).
113. Lerman, S. J., Kucera, J. C. \& Brunken, J. M. Nasopharyngeal carriage of antibiotic-resistant Haemophilus influenzae in healthy children. Pediatrics 64, 287-291 (1979).

114. Murphy, T. F. et al. Nontypeable Haemophilus influenzae as a pathogen in children. Pediatr. Infect. Dis. J. 28, 43-48 (2009).

115. Dagan, R. \& Klugman, K. P. Impact of conjugate pneumococcal vaccines on antibiotic resistance. Lancet Infect. Dis. 8, 785-795 (2008).

116. Wilby, K. J. \& Werry, D. A review of the effect of immunization programs on antimicrobial utilization. Vaccine 30, 6509-6514 (2012).

117. Leach, A. J. et al. Reduced middle ear infection with non-typeable Haemophilus influenzae, but not Streptococcus pneumoniae, after transition to 10 -valent pneumococcal non-typeable $H$. influenzae protein D conjugate vaccine. BMC Pediatr. 15, 162 (2015).

118. Simpson, J. L. et al. Airway dysbiosis: Haemophilus influenzae and Tropheryma in poorly controlled asthma. Eur. Respir. J. 47, 792-800 (2015).

119. Zhang, Q. et al. Airway microbiota in severe asthma and relationship to asthma severity and phenotypes. PLOS ONE 11, e0152724 (2016).

120. Johansen, H. K. \& Gotzsche, P. C. Vaccines for preventing infection with Pseudomonas aeruginosa in cystic fibrosis. Cochrane Database Syst. Rev. 8, CD001399 (2015)

121. Maiden, M. C. Population genomics: diversity and virulence in the Neisseria. Curr Opin. Microbiol. 11 467-471 (2008)

122. Rao, D., Webb, J. S. \& Kjelleberg, S. Microbial colonization and competition on the marine alga Ulva australis. Appl. Environ. Microbiol. 72, 5547-5555 (2006).

123. Ley, R. E., Peterson, D. A. \& Gordon, J. I. Ecological and evolutionary forces shaping microbial diversity in the human intestine. Cell 124, 837-848 (2006)

124. Comas, I. et al. Out-of-Africa migration and Neolithic coexpansion of Mycobacterium tuberculosis with modern humans. Nat. Genet. 45, 1176-1182 (2013)

125. Walker, T. M. et al. Whole-genome sequencing to delineate Mycobacterium tuberculosis outbreaks: a retrospective observational study. Lancet Infect. Dis. 13, 137-146 (2013).

126. Snitkin, E. S. et al. Tracking a hospital outbreak of carbapenem-resistant Klebsiella pneumoniae with whole-genome sequencing. Sci. Transl Med. 4 148ra116 (2012)

127. LiPuma, J. J., Dasen, S. E., Nielson, D. W., Stern, R. C. \& Stull, T. L. Person-to-person transmission of Pseudomonas cepacia between patients with cystic fibrosis. Lancet 336, 1094-1096 (1990).
128. Cheng K et al. Spread of $\beta$-lactam-resistant Pseudomonas aeruginosa in a cystic fibrosis clinic. Lancet 348, 639-642 (1996).

129. Bryant, J. M. et al. Whole-genome sequencing to identify transmission of Mycobacterium abscessus between patients with cystic fibrosis: a retrospective cohort study. Lancet 381, 1551-1560 (2013).

130. Bryant, J. M. et al. Emergence and spread of a human transmissible multidrug-resistant nontuberculous mycobacterium. Science 354, 751-757 (2016).

131. Saiman, L. et al. Infection prevention and control guideline for cystic fibrosis: 2013 update. Infect. Control Hosp. Epidemiol. 35 (Suppl. 1), S1-S67 (2014).

132. Chotirmall, S. H. \& McElvaney, N. G. Fungi in the cystic fibrosis lung: bystanders or pathogens? Int. J. Biochem. Cell Biol. 52, 161-173 (2014).

133. Parize, P. et al. Impact of Scedosporium apiospermum complex seroprevalence in patients with cystic fibrosis. J. Cyst. Fibros. 13, 667-673 (2014).

134. Larkin, E. et al. The emerging pathogen Candida auris: growth phenotype, virulence factors, activity of antifungals, and effect of SCY-078, a novel glucan synthesis inhibitor, on growth morphology and biofilm formation. Antimicrob. Agents Chemother. 61 e02396-16 (2017)

135. de Wit, R. \& Bouvier, T. 'Everything is everywhere, but the environment selects'; what did Baas Becking and Beijerinck really say? Environ. Microbiol. 8, 755-758 (2006).

136. Byrd, A. L. \& Segre, J. A. Infectious disease. Adapting Koch's postulates. Science 351, 224-226 (2016).

Acknowledgements

W.O.C.M.C., M.J.C. and M.F.M. receive funding from the Wellcome Trust and from the Asmarley Trust. W.O.C.M.C. and M.F.M. are joint Wellcome Senior Investigators. W.O.C.M.C. also received funding for microbiome studies with a Senior Investigator award from the National Institute for Health Research, United Kingdom.

\section{Author contributions}

W.O.C.M.C. carried out research, wrote the article and contributed to discussions, review and editing of the manuscript. M.J.C. and M.F.M. carried out research and contributed to discussions, review and editing of the manuscript. The authors gratefully acknowledge the considerable input of two anonymous reviewers.

\section{Competing interests statement}

The authors declare no competing interests.

Publisher's note

Springer Nature remains neutral with regard to jurisdictional claims in published maps and institutional affiliations.

\section{ERRATUM \\ Inserting proteins into the bacterial cytoplasmic membrane using the $\mathrm{Sec}$ and YidC translocases}

Kun Xie and Ross E. Dalbey

Nature Reviews Microbiology 6, 234-244 (2008)

This article was published with an incorrect DOI. The correct DOI is $10.1038 / \mathrm{nrmicro3595}$. This has now been corrected in

the online version. We apologize to the authors and to readers for any confusion caused.

\section{CORRIGENDUM}

\section{Archaea and the origin of eukaryotes}

Laura Eme, Anja Spang, Jonathan Lombard, Courtney W. Stairs and Thijs J. G. Ettema

Nature Reviews Microbiology 15, 711-723 (2017)

On pages 714-715 of this article, in the first paragraph of the section What do we currently know about LECA?, the sentence "Phylogenomic and comparative genomic analyses have led to the hypothesis that LECA, estimated to have lived 1-1.9 million years ag $^{54}$, already was a fully fledged eukaryote and possessed a large number of features that are uniquely found in modern eukaryotes ${ }^{55,56}$." should have read "Phylogenomic and comparative genomic analyses have led to the hypothesis that LECA, estimated to have lived $~ 1-1.9$ billion years ago ${ }^{54}$, already was a fully fledged eukaryote and possessed a large number of features that are uniquely found in modern eukaryotes ${ }^{5,56}$." This has been corrected in the online version of the article. The authors apologize to the readers for any misunderstanding caused. 